\title{
Collective Behavior of Equity Returns and Market Volatility
}

\author{
Zhaoyuan $\mathrm{Li}^{1}$, Sibo Liu ${ }^{2}$ and Maozai Tian ${ }^{31}$ \\ ${ }^{1}$ Department of Statistics and Actuarial Science, the University of Hong Kong \\ ${ }^{2}$ Department of Finance and Insurance Lingnan University \\ ${ }^{3}$ Center for Applied Statistics, School of Statistics Renmin University of China
}

\begin{abstract}
We analyze the cross-correlation between logarithmic returns of 1108 stocks listed on the Shanghai and Shenzhen Stock Exchange of China in the period 2005 to 2010. The results suggest that the estimated distribution of correlation coefficients is right shifted in the tumble time of Chinese stock market. Due to the large share of maximum eigenvalue, the principal correlation component in Chinese stock market is dominant and other components only have trivial effects on the market condition. The same-signed corresponding vector elements enable us to propose the maximum eigenvalue series as an indicator for collective behavior in the equity market. We provide the evidence that the largest eigenvalue series can be used as an effective indicative parameter to describe the collective behavior of stock returns, which is found to be positively correlated to market volatility. By using time-varying windows, we find the positive correlation diminishes when the market volatility reaches both highest and lowest level. By defining a stability rate, we display that the collective behavior of stocks tends to be more homogeneous in the context of crisis than the regular time. This study has implications for the arising discussions on correlation risk.
\end{abstract}

Key words: cross-correlation, collective behavior, financial volatility, correlation risk.

\section{Introduction}

As representative complex systems, financial markets are organized by various unexpected phenomena according to non-trivial interactions among heterogeneous agents. Recently, more and more methodological tools are introduced from physicsto economics and finance. A new subject, as called "Econophysics", is developed. This interdisciplinary field is pioneered by thegroundbreaking work by Mantegna and Stanley(1999), Dragulescu and Yakovenko (2000), Sornette (2002), Bouchaud and Potters (2003) and etc.

This paper focuses on the collective behavior of equities in the stock market using the pairwise correlation matrix of individual equities. The correlation between equities is important

\footnotetext{
${ }^{1}$ Corresponding author.
} 
for both investment strategy and risk management (Driessen, Maenhout and Vilkov, 2013). In the turbulent time of financial crisis, in particular, the collective behavior of stocks in the market is different from usual conditions. In economics and finance, traditional Markowitz's theory of optimal portfolios has the strict hypothesis of combination normal distribution with constant correlation between each asset (Buraschi, PorchiaandTrojani, 2010). In the recent literature, the importance of taking into account the time-variation of correlations is highlighted by Aneja, et al. (1989), Chesnay and Jondeau(2001), and Jea, et al. (2005). These authors estimate the average correlation coefficient of a group of stocks in the portfolio analysis. Other researchers focus on the study of co-movement of international indexes as well as stocks within a market, see Agmon (1972), Polleta and Wilson (2006), Arouria, et al. (2006), and Bekaert, et al. (2009). The correlations between international equity markets are investigated to determine whether they would increase during periods of high volatility. However, the existing literatures in economics mostly concentrate on the pairwise correlation between two indexes or stocks. Few consider all the individual stocks in a market and analyze the time-variation of the collective phenomenon.

In physics, researchers explore this issue in a different perspective, see Laloux, et al. (2000), Sharifi, et al. (2004), Petersen, et al. (2010), Podobnik, et al. (2007, 2009, 2010), and Plerou, et al. (2000, 2001). Most of the literature in physics applies Random Matrix Theory (RMT) on an empirically measured financial correlation matrix, which examines the sensitivity of the spectral properties of a random matrix. Other researchers concentrate on the long-range powerlaw autocorrelations in the correlation matrix. Liu, et al. (2011) and Tse, et al. (2010) study the cross correlation matrix in the network perspective, and some properties about stock network constructed are found. For example, Liu, et al. (2011) suggest the level of resemblance of the scale-free structure of the stock network (indicated by the fitting error of the power-law exponent) is an indicator of the normality of the market. However, these studies do not consider the time varying pattern of correlation distribution. According to the discussion of Liu, et al. (2011) and Tse, et al. (2010), stocks whose correlation coefficients higher than a constant threshold are connected. And more edges will weaken the scale-freeness of the degree distribution. Our results in this paper show that in the time of fierce volatility, the distribution of correlation matrix elements are is right shifted, which naturally brings more edges in the network and leads to the so-called disrupted scale-freeness. The evidence in this paper exhibits the fundamental reason and underlying mechanism for the pattern observed in prior studies. And we suggest that the statistical properties of equit returns is necessary before employing new approach in the data analysis.

Maskawa, et al. (2009) suggest the probability of large price movement conditional on the maximum eigenvalue can be taken as a measure of risk. In this paper, we propose a similar but more delicate approach to analyze the cross-correlation of stock returns in the Chinese stock market. By diagonalization, we obtain the eigenvalue and eigenvector series from the correlation matrix. Eigenvalues indicate the proportions of different independent correlation components in the markets. The element in the eigenvectors corresponds to the contributions of each individual stock. The magnitude of eigenvector elements thus denotes the contribution of each stock to a certain independent market factor. 
Using the real data in China, we find that the maximum eigenvalue accounts a large share of matrix trace and the elements of the eigenvector according to the maximum eigen-value are mostly same-signed. In this regard, the principal correlation component in the Chinese market is dominant. Thus, the maximum eigenvalue can be taken as an indicator for the degree of collective behavior. In the turbulent time of bear market, the maximum eigenvalues reach the record-high level. The Spearman correlation coefficients between market volatility and maximum eigenvalue series are all significantly positive across different windows. But the positive correlation effect weakens in the context of highest and lowest level of market volatility. The trough of varying correlation between market volatility and collective behavior indicator enable us to describe the changes of market risk. By defining a stability rate, we find the collective behavior of stocks tends to be more homogeneous in crisis than usual time. The evidence suggests the correlation risk is very important in understanding the mechanism of stock co-movement. Investment strategy and pricing method accounting correlation risk need to be further explored according to our study.

The article is organized as follows. Section 2 describes the data set we use and briefly introduces the methodology. In Section 3, we analyze the data and interpret the results. Section 4 concludes.

\section{Data and Methodology}

We analyze 1108 time series with 1457 daily records in the 6-year period, 4 January 2005 to 31 December 2010. 1108 individual equities cover most of the stocks listed before 2005 and traded in the "A" shares of Shanghai and Shenzhen stock exchange. The logarithmic change ("returns") will be considered for further discussion. It is defined as

$$
R_{i}(t)=\log \left(P_{i}(t)\right)-\log \left(P_{i}(t-1)\right),
$$

where $P_{i}(t)$ denotes the price of individual stock $i$ at time $t$. We believe that the logarithmic change is a suitable measure for reflecting the performance of investors, which is directly related to the investment strategy and risk management.

To quantify the linear relationship between individual stocks, we apply the general correlation coefficient as follows. Consider the $\mathrm{N}$-variable time series $X=\left\{X_{i}(t): i=\right.$ $1, \ldots, N ; t=1, \ldots, T\}, i$ denotes different individual stocks we choose, $t$ is the time period. The correlation matrix for the time series is

$$
\operatorname{corr}_{i j}(t)=\frac{\operatorname{Cov}_{i t}(t)}{\sigma_{i} \sigma_{j}}
$$

where $\operatorname{Cov}_{i j}(t)=<X_{i}(t) X_{j}(t)>-<X_{i}(t)><X_{j}(t)>, \sigma_{i}=\sqrt{<X_{i}^{2}>-<X_{i}>^{2}}$ is the standard deviation of $X_{i} . X_{i}(t)$ and $X_{j}(t)$ denote price returns $R(t)$ of stock $i$ and stock $j$, respectively, in the period $t=1$ to $T$. For correlation matrix, the elements $\operatorname{corr}_{i j}$ are restricted to the domain $[-1,1]$, where $\operatorname{corr}_{i j}=1$ corresponds to perfect positive correlations, $\operatorname{corr}_{i j}=-1$ 
corresponds to perfect negative correlations, and $\operatorname{corr}_{i j}=0$ corresponds to insignificant correlations of stock pairs.

We apply the diagonalization of correlation matrix and differentiate the independent market factors. The diagonalization of $N \times N$ symmetric matrix $C$, denoting matrix series $\operatorname{corr}_{i j}(t)$, can be briefly explained as follows

then,

$$
P^{-1} C P=\operatorname{diag}\left(\lambda_{1}, \lambda_{2}, \ldots, \lambda_{N}\right)
$$

Write $P$ as a block matrix of its column vectors

or

$$
P=\left(\vec{a}_{1}, \vec{a}_{2}, \cdots, \vec{a}_{N}\right) \text {, }
$$

$$
C \vec{a}_{i}=\lambda_{i} \vec{a}_{i}, i=1,2, \ldots, N .
$$

The column vectors of $P$ are eigenvectors of $C$, and the corresponding diagonal entry $\lambda_{i}$ is the corresponding eigenvalue. The eigenvalues denote the different importance of components for the tendency in the market. The invertibility of $P$ determines that the eigenvectors are linearly independent. So through diagonalization, independent correlation components within a system are obtained. Moreover, the distribution of eigenvalues contains more information of components. Each element in the eigenvector denotes the contribution of a certain individual stock and the positive or negative sign of the element reflects the direction of the contribution and the magnitude denotes the degree of the contribution. In addition, the turnover rate of the individual stocks within the peak zone of the eigenvector through the time can reflect the stability of the collective behavior.

The market return evolvement is expressed by average return of market index, which is defined as

$$
A V I(t)=\frac{1}{k \Delta t} \sum_{i=0}^{k \Delta t} I(t i)
$$

where $\Delta t$ denotes the step of the window that we calculate the cross-correlation matrix and also the window we use to calculate the standard deviation of market index. In our case, the index we applies is Shanghai Stock Exchange Composite Index (SCI), the key index of Chinese stock market, and $I(t i)$ is the logarithmic return of SCI. And the $\Delta t$ is one day, thus $k \Delta t$ is the width of the window. In order to measure the volatility of the market, we define the standard deviation of index as follows

$$
\operatorname{StdI}(t)=\sqrt{\frac{1}{k \Delta t-1} \sum_{i=0}^{k \Delta t}\left[\left(I\left(t_{i}\right)-<I(t)>\right)^{2}\right]},
$$

To identify whether the correlation exists between the collective behavior indicator and market volatility, Spearman's rho statistic is introduced, which is a measure of rank correlation in terms of the integral transforms of two series, $X$ and $Y$, formulated as

$$
\rho_{s}=\frac{12}{n\left(n^{2}-1\right)} \sum_{i=1}^{n}\left(\operatorname{rank}\left(x_{i}\right)-\frac{n+1}{2}\right)\left(\operatorname{rank}\left(y_{i}\right)-\frac{n+1}{2}\right) \text {. }
$$


Here $x$ is the market volatility, i.e. StdI, and $y$ is the maximum eigenvalue series.

\section{Results}

\subsection{Evolvement of correlation matrix and eigenvalue spectrum}

First we discuss the shifted distribution of cross correlation matrix elements. We plot the evolvement of correlation matrix distribution with 30 days moving window $(k=30)$ and 30-day step. Figure 1 is the top view of the time-varying kernel density of correlation coefficients. Each curve in the figure denotes correlation coefficient distribution of 30days. The red curves denote the correlation distribution in the tumble time of Chinese stock market from December 2007 to October 2008. The distributions of correlation coefficients are significantly right shifted in the crisis representing a strong collective behavior of individual stocks. The side view of the evolvement of correlation matrix is plotted in Figure 2 that also clearly shows this pattern.

In order to further analyze the independent correlation factors in the market, we examine time-varying feature of the proportion of maximum eigenvalue and other eigenvalue to the trace. We report the spectrum of eigenvalue in the whole period in Figure 3 as many researchers do. We find that basically the maximum eigenvalues hold largest proportion of trace. The principal component denoted by the maximum eigenvalue is dominant. In other words, the maximum eigenvalue accounts a large proportion of trace, while other market factors denoted by other eigenvalues are less important. This graphical pattern is consistent with existing literature, but our interpretation is rather different. Finding a similar distribution shape as in Figure 3, Podobnik, et al. (2001, 2007, 2009, 2010) introduce random matrix theory (RMT) to filter the randomness of cross-correlation matrix. We believe in contrast the analysis of trivial market factors indicated by small eigenvalues is not convincing. The maximum eigenvalue holds the most important information of equities correlation in the market. Before adopting maximum eigenvalue as a collective behavior measurement, we need a close examination of the eigenvectors corresponding to eigenvalues.

As we discuss above, each element in the eigenvector corresponds to each individual stock and the magnitude of element denotes the contribution of each stock to the market correlation proportion indicated by eigenvalues. The distributions of eigenvectors elements are plotted in Figure 4. 


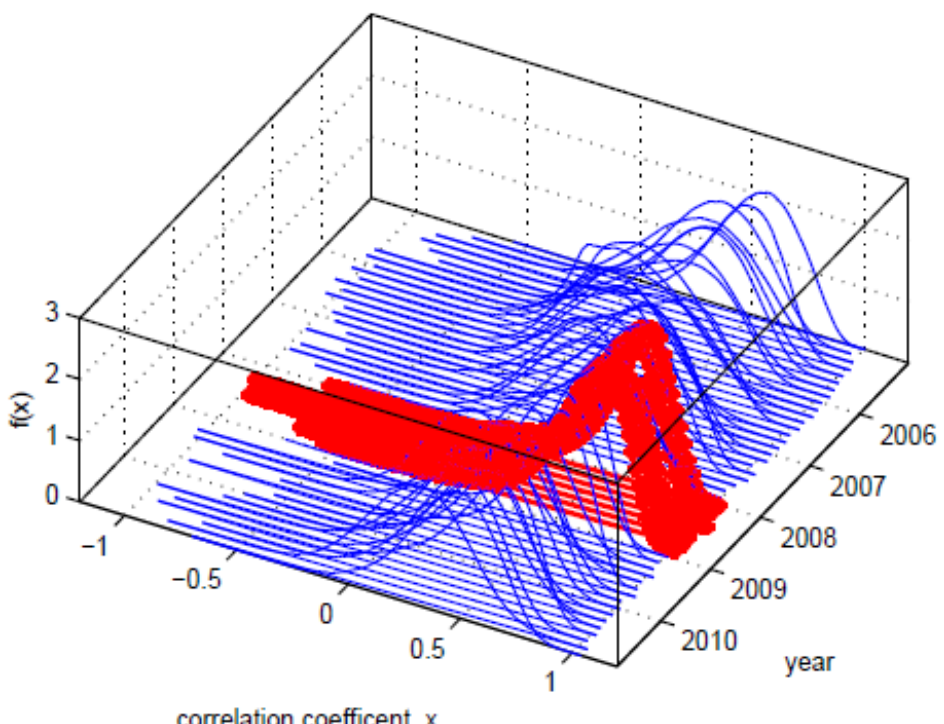

Figure 1: The evolvement of correlation matrix distribution (top view). With 30 days moving window and 30-day step, the top view of the kernel density of correlation coefficients through the time is plotted. Each curve in the figure denotes correlation coefficient distribution of 30 days. The red curves denote the correlation distribution in the tumble time of Chinese stock market from December 2007 to October 2008. The distributions of correlation coefficients are significantly right shifted in the time of crisis representing a strong co-movement of individual stocks.

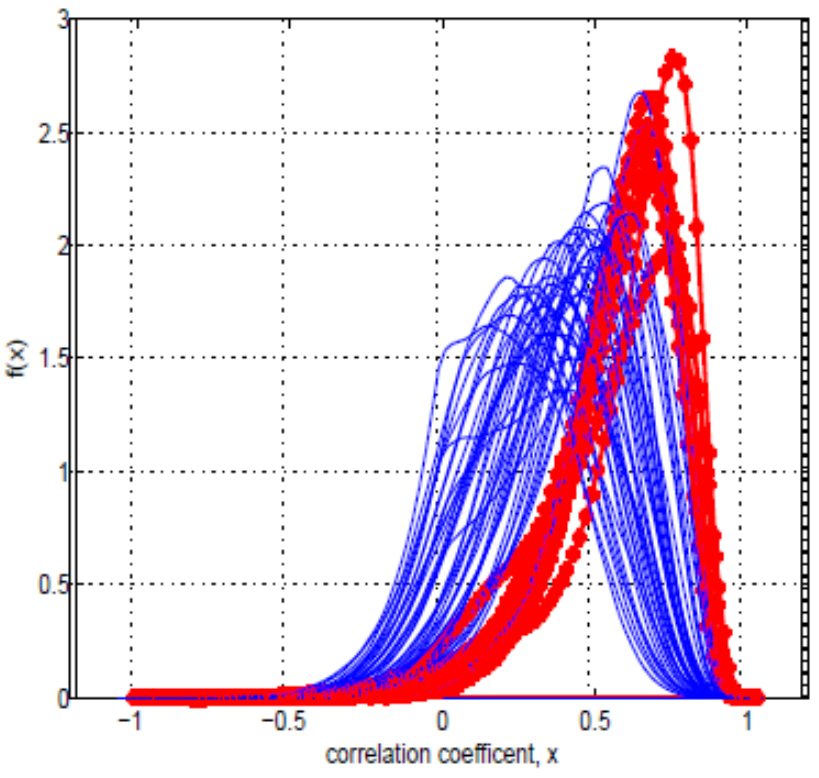

Figure 2: The evolvement of correlation matrix distribution (side view). The side view of the kernel density of correlation coefficients through the time is plotted. The right-shifted distributions of correlation coefficients in the financial crisis are clearly observed. 


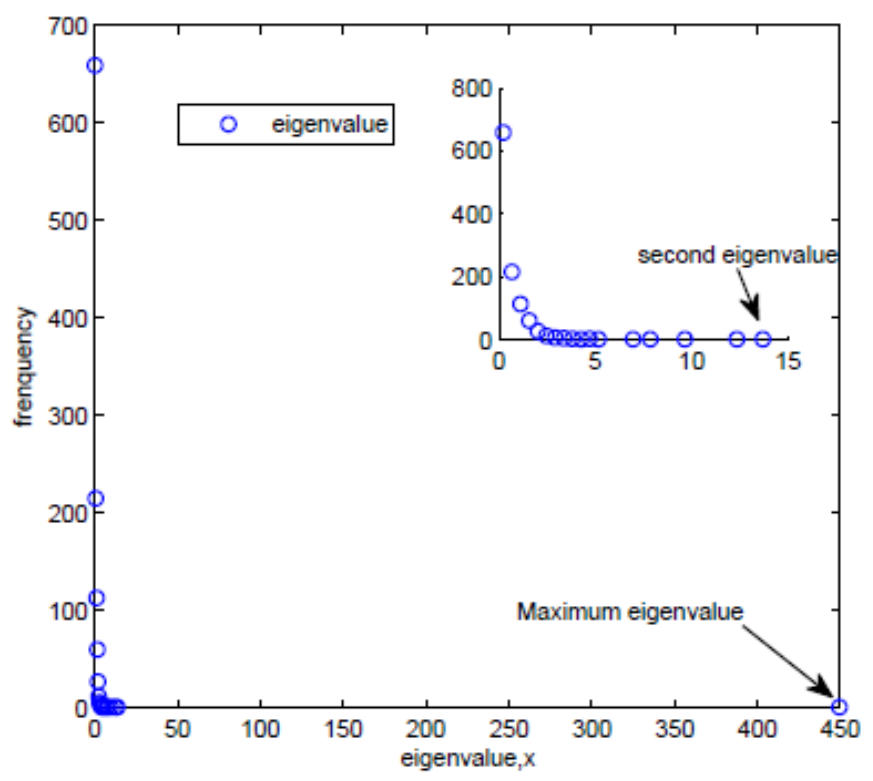

Figure 3: The distribution of eigenvalues of correlation matrix in the whole period. The maximum accounts the most of the share of trace, and other small eigenvalues denotes trivial factors in the market.

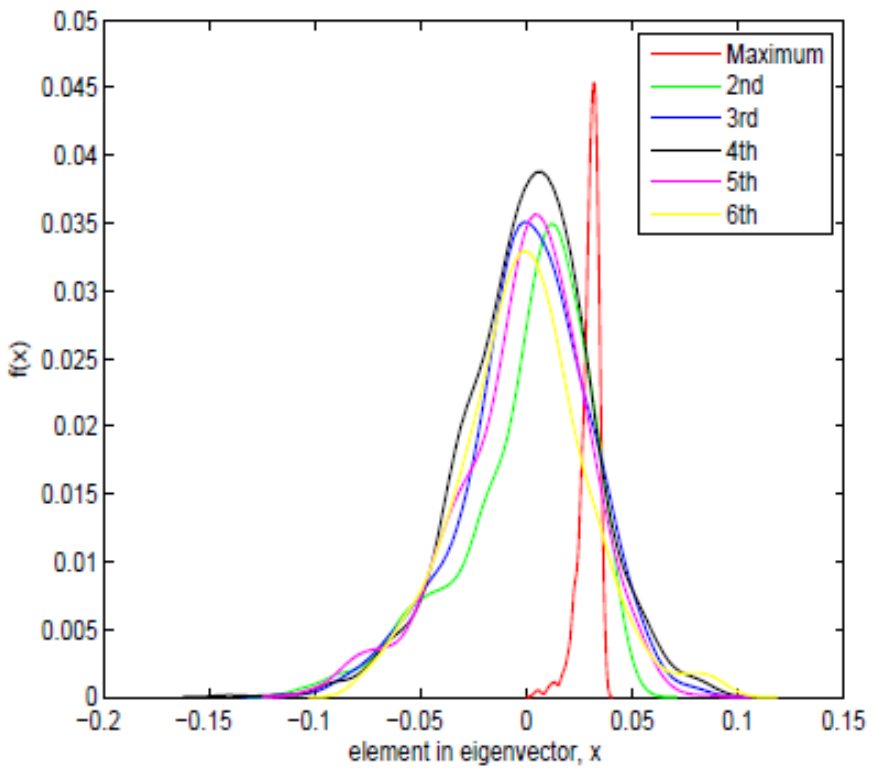

Figure 4: Kernel density distribution of eigenvector elements according to corresponding eigenvalues in the whole period. Vertical axis is the probability density function. In the whole period, the overwhelming majority of the elements in the maximum eigenvalue is positive, while for other eigenvectors the elements have mixed signs. In other words, for the principal component of correlation matrix, the 
elements of corresponding vector representing individual equities are on the same direction. Thus, the maximum eigenvalue, as the same fluctuated market correlation component, is appropriate to reflect the collective behavior.
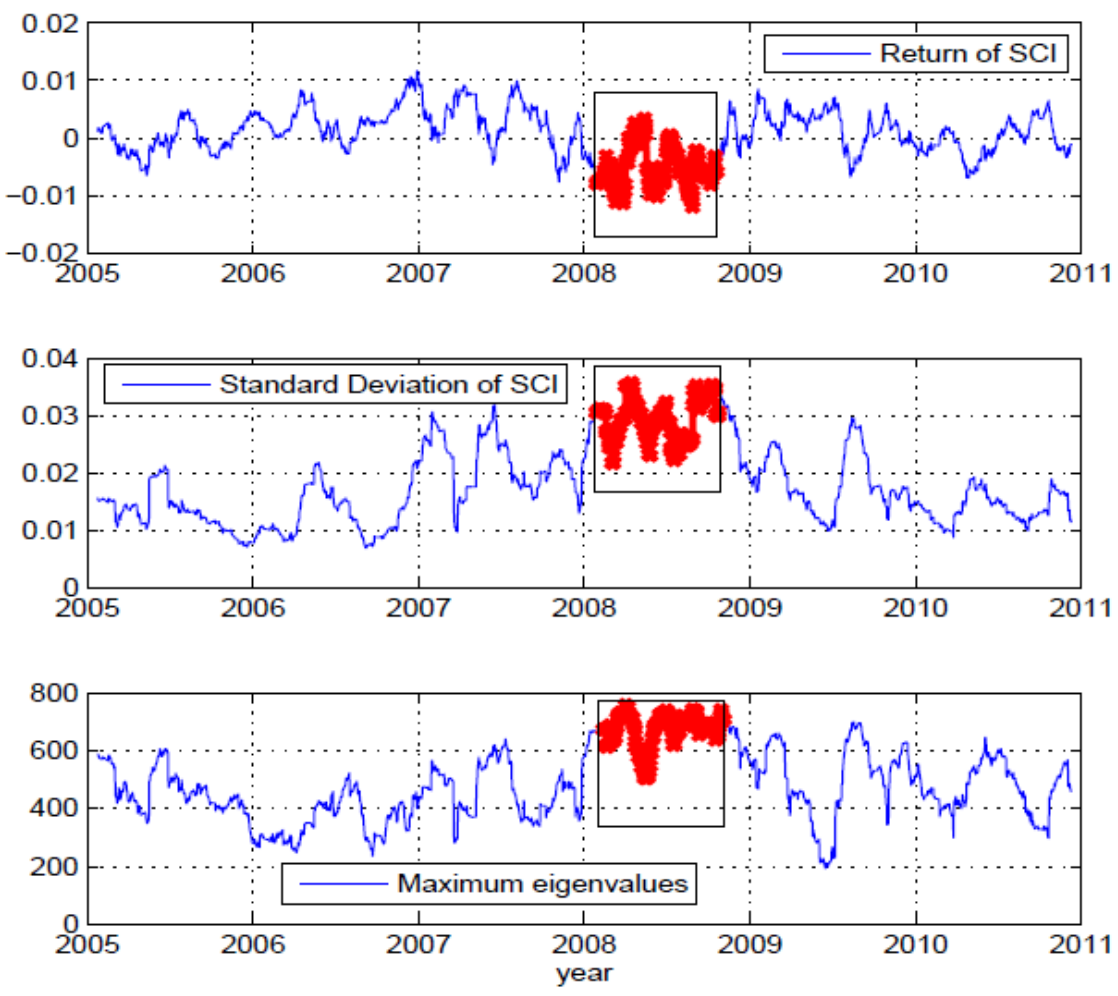

Figure 5: The evolvement of average return, standard deviation of SCI and the maximum eigenvalue series. The maximum eigenvalue series are negatively related to return of SCI and positively related to standard deviation of SCI, with sharp spikes well matched. Both the maximum eigenvalue and volatility indicator (standard deviation of SCI) reach the historical high level (highlighted by red line and solid rectangle) in the tumble time of Chinese stock market.

Figure 4 shows that compared with other eigenvectors, the overwhelming majority of vector elements responding maximum eigenvalue is positive. For the principal component of correlation matrix, the elements of corresponding vector representing individual equities are on the same direction. According to the above discussion, the proportions of eigenvalues, except for maximum eigenvalue, are rather small and reflect the trivial factor of market equity correlation. Maximum eigenvalue, as the same fluctuated market correlation proportion, is appropriate to quantify the collective behavior effect.

\subsection{Correlation between market volatility and collective behavior}


With 30-days moving window $(\mathrm{k}=30)$ as in Figure 1 and 2, we adjust the step to one day for further analysis. The evolvement of average return and standard deviation of SCI as well as the maximum eigenvalue series is displayed in Figure 5. On the whole, the maximum eigenvalue series are negatively related to return of SCI and positively related to standard deviation of SCI, with sharp spikes well matched. Both the maximum eigenvalue and volatility indicator (standard deviation of SCI) reach the historical high level (highlighted by red line and solid rectangle) in the tumble time of Chinese stock market. The collective behavior of equites has been strengthened in the turbulence of financial crisis.

Because market volatility directly reflects the risk level in the portfolio analysis, we next examine whether the correlation really exists between market volatility and collective behavior indicator (the maximum eigenvalue series). Market volatility and collective behavior indicator are regarded as explanatory variable and response variable, respectively. KolmogorovSmirnovtest (Struart, et al., 1999, Goldstein, et al., 2004) shows that distributions of both the explanatory and response variables deviate from normality. Hence, the correlation between explanatory and response variables should be determined by rank correlation coefficient, and here we adopt Spearman's correlation coefficient (Hollander and Wolfe, 1973), test settings of which are as follows.

- Null hypothesis $H_{0}: \rho=0$ which denotes there is no significant correlation between explanatory variable and response variable.

- Alternative hypothesis $H_{1}: \rho \neq 0$. the alternative hypothesis is accepted if the statistic is larger than the critical value.

Here we use the significance level $\alpha=0.001$. We calculate the maximum eigenvalue and market volatility series in the circumstances of different window ranging from one week to two years. At each window width, Spearman's correlation coefficient is calculated and tested. The results reported in Figure 6 show that there are significant correlations across all range of windows. Figure 6 displays a clear pattern that correlation effect between market volatility and maximum eigenvalue increase with a decelerating speed as the window widens. At the 1.5 -year window width the correlation reaches the highest point, while in the circumstance of window wider than 1.5 year, correlation between market volatility and collective behavior indicator inversely weakens. We believe that due to the fact that wider windows actually average out the trivial information and focus on the long term pattern, correlation effect is more clearly shown. However, extremely wide window will average out essential information and reduce the observed correlation coefficient. Hence, the evidence suggests that the correlation between market volatility and collective behavior indicator exists and the degree of correlation increases as the observation window widens. The correlation risk generated by the collective behavior may play a vital role in portfolio strategy, especially in the crisis period.

\subsection{Correlation between market volatility and collective behavior through the time}


In this subsection, we examine whether correlation between market volatility and collective behavior evolves through the time, a neutral extension of the analysis in the last subsection. We apply the moving window method to reflect the time variation feature of the relationship between market volatility and collective behavior. Figure 7 displays market index volatility and the varying Spearman's correlation between market volatility and collective behavior indicator. Basically there are two troughes for varying correlation. First is around 2006 the historical low point of market volatility and second is around financial crisis the highest level of market risk. In general, the correlation between market volatility and collective behavior indicator becomes weak at the inverse point of market risk. In other words, the trough of varying correlation between market volatility and collective behavior indicator enable us to describe the changes of market risk.

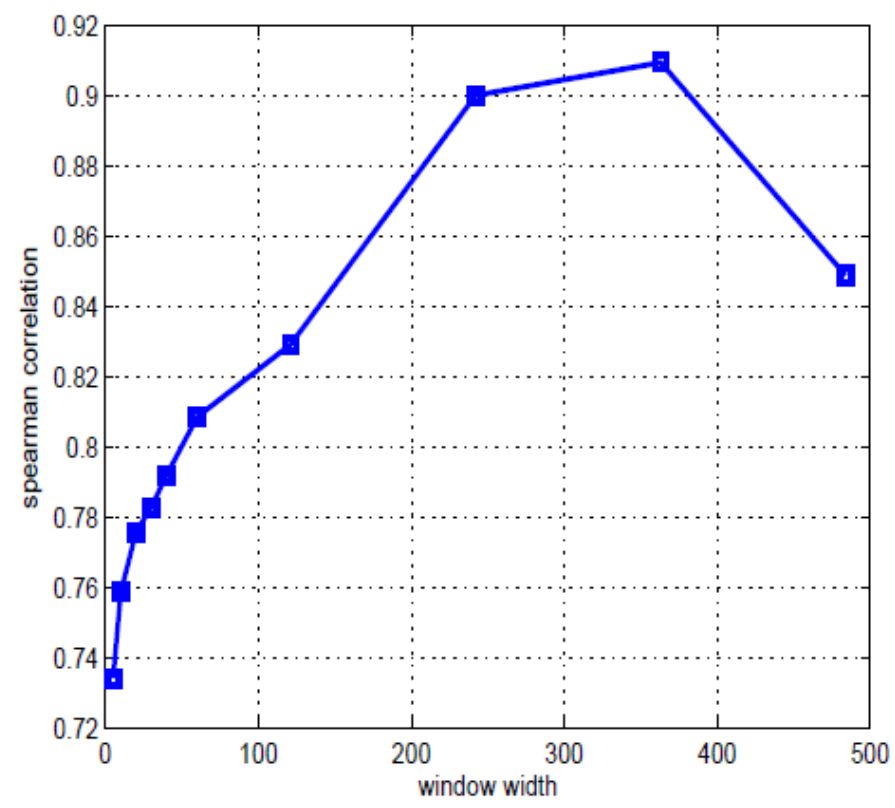

Figure 6: Spearman's correlation coefficient across different windows. In the circumstances of different window ranging from one week to two years, the correlation matrixes are constructed and the eigenvalue series and market volatility indicator are calculated for Spearman's correlation coefficient. The correlation coefficients are significant across all range of windows at the 0.001 level. The degree of correlation increases as the width of window broadens, while at 1.5 year window reaches the highest level. 

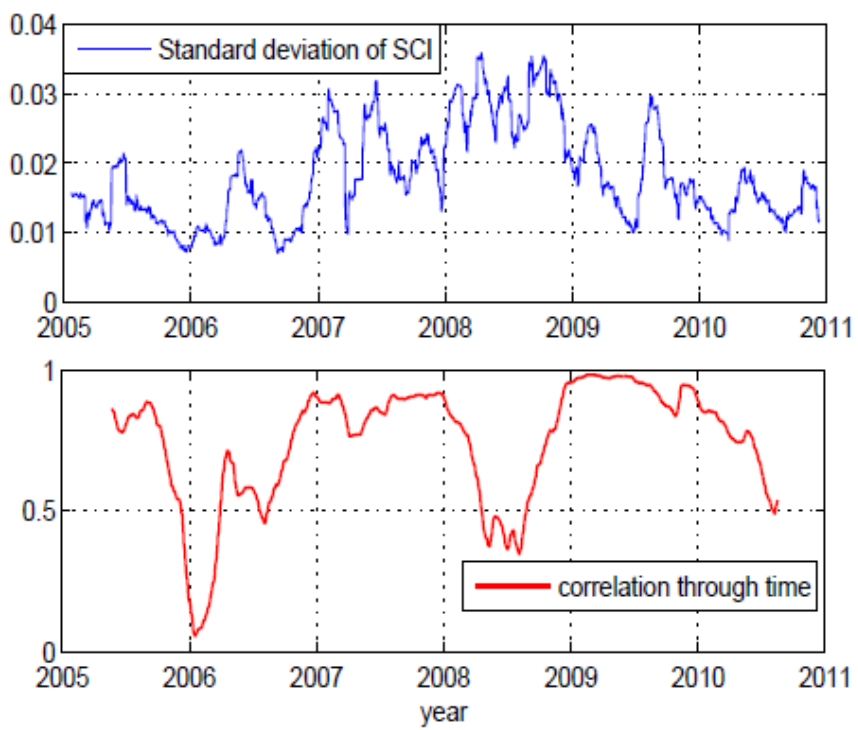

Figure 7: Correlation between market volatility and collective behavior through the time. The varying Spearman's correlation between market volatility and collective behavior indicator weakens in 2006 the lowest point of market volatility and 2008, the highest level of market risk. The trough of varying correlation between market volatility and collective behavior indicator tends to coincide with inverse point of market risk trend.

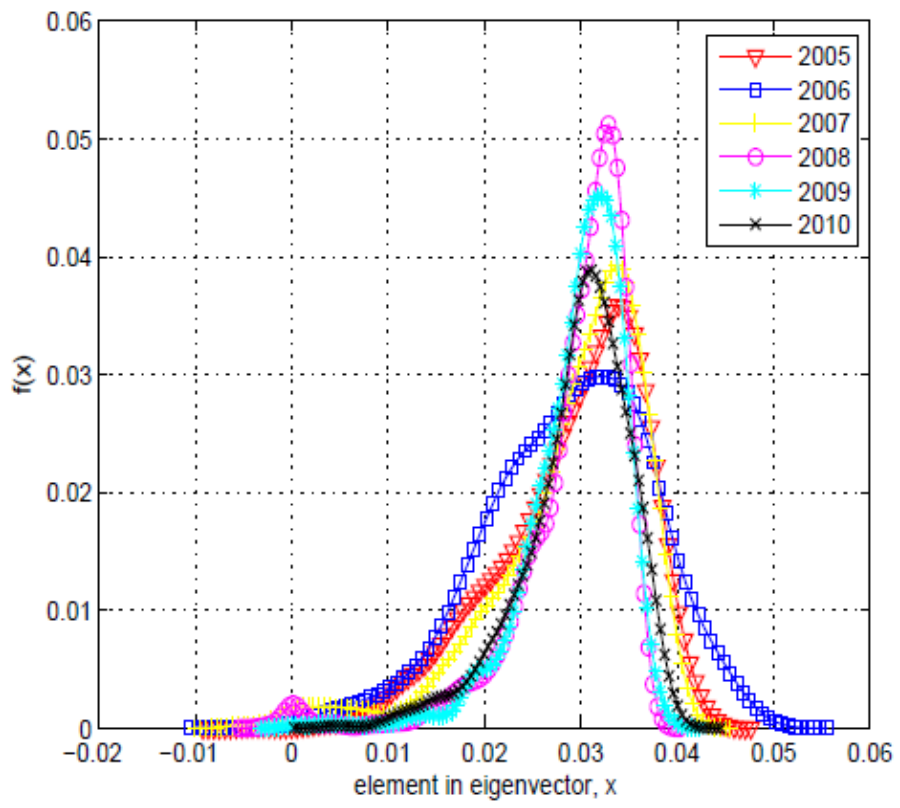

Figure 8: Distributions of elements in eigenvector corresponding to Maximum eigenvalue. In 2006, the distribution is less peaked than other years, while in 2008 , eigenvector distribution is the most peaked 
indicating the concentrated contributions from individual stocks. In other words, in the market anomalies period of Chinese stock market, the magnitudes of contribution from equites are clustered within a narrow area.

Here we point out that correlation between market volatility and collective behavior varying through the time does not conflict with the pattern that collective behavior co-moves with market volatility. Although the degree of correlation weakens at the lowest and highest level of volatility, the co-movement relationship is still significant through the time. And the analysis of time variation feature also confirms that market volatility strengthens collective behavior of equity returns.

\subsection{Distribution of elements in eigenvectors and stability indicator}

Two facts need to be highlighted again. Each individual equity is represented by element of eigenvectors. And elements of vector corresponding to maximum eigenvalue are same-signed. This pattern suggests equities contribute to principle correlation proportion on the same direction. However, in terms of other stock factors, the contribution of stock correlation is not on the same direction. This is the reason why we adopt the maximum eigenvalue as the indicator. Next, we analyze the turnover of individual equites contribution to principle correlation proportion in different periods to reflect the stability of the system in the context of the collective behavior.

The distributions of elements of vectors corresponding to maximum eigenvalues through years are plotted in Figure 8. In 2006 the distribution is less peaked than other years, while in 2008, the eigenvector distribution is most peaked indicating concentrated contributions from

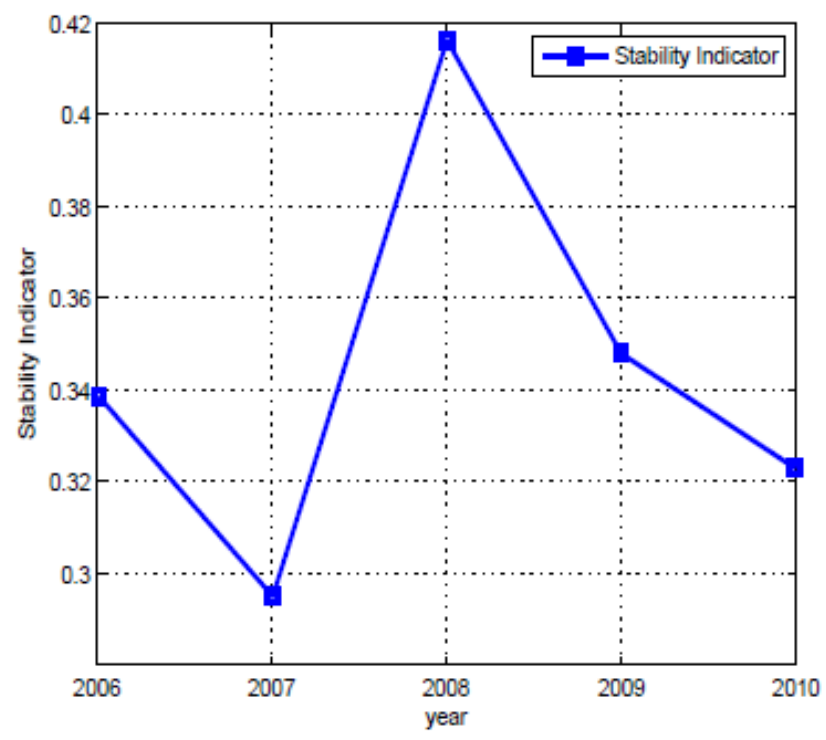

Figure 9: Evolvement of stability indicator. Defined as the number of stocks from the peak at time $(t-1)$ divided by the number of stocks in the peak at time $t, S_{t}$ reaches the highest level in financial crisis period of 2008. Thus, the collective behavior is more homogeneous in the tumble time of market. 
individual stocks. In other words, in the crisis period of Chinese stock market, the magnitudes of contribution from equites are concentrated within a narrow area. In order to capture the stability of stock system in the context of collective behavior, we analyze the turnover rate of vector elements in the peak area through the years. The kurtosis of distribution varies through years. To hold the same number of equities within the peak area, we consider $30 \%$ of the equites in the neighborhood of peak value. The stability rate is defined as follows.

$$
S_{t}=\frac{n_{t}-1}{N_{t}}
$$

where $N_{t}$ denotes the number of stocks in the peak at time $t$. In our case, $N_{t}$ is $332,30 \%$ of all individual stocks. $n_{t}-1$ is the number of equities in the peak area at time $t$ also in thepeak at time $(t-1)$. Thus $S_{t}$ reflects the turnover of vector elements in the peak area through the years. Higher $S_{t}$ indicates higher degree of the stability of stock system evolvement. In Figure 9, we report the stability rate.

The stability rate clearly shows that in the tumble time of Chinese stock market, the stability of stocks reaches the highest point, while in regular time the homogeneity is slightly weak. In this regard, in the context of equities collective behavior in financial crisis, the elements of vector have less turnover rate through the years. The stock system in the tumble time of crisis tend to be moving on the same direction. According to the discussion above, the market volatility is positively correlated to the collective behavior indicator and the stability indicator is generally accorded with this pattern. Therefore, fierce volatility strengthes collective behavior and the homogeneity of individual equities movement.

\section{Conclusion}

In this paper, through detailed eigen-analysis of correlation matrix, we find several interesting statistical properties of Chinese stock market. The key finding is that fierce volatility strengthens the collective behavior of equity returns. We present the results and related intuitions for investment strategy in the following statement.

First, the estimated distributions of correlation coefficients are right shifted in the tumble time of Chinese stock market. Stock market crashes stimulate high correlations among financial equities, which is consistent with many empirical results of existing literature. For portfolio managers and financial regulators, this statistical pattern is of great importance. As highlighted above, the combination normal distribution with constant correlation between each asset is an indispensable hypothesis of traditional Markowitz's theory of optimal portfolios (Buraschi, Porchiaand Trojani, 2010). In real financial markets, however, the correlations are time-varying. It leads to a concentration of so called "correlation risk". As confirmed by our analysis, during periods of market turbulence, correlations significantly increase and risk adjusted allocation of assets with a focus on "correlation risk" in stock crashes will be necessary.

Second, the maximum eigenvalue takes dominant share of the trace. The evidence reflects that the principle correlation component for Chinese stock market plays a vital role. Other than 
analyzing the spectrum of eigenvalues through general applied random matrix theory (RMT), we focus on the statistical properties of correlation matrix and large eigenvalues. We find that the maximum eigenvalue takes large share of the trace indicating the dominant market factor, while other eigenvalues which take small share of the trace can be considered as trivial factors of the market. In this sense, the spectrum analysis of eigenvalues may provide limited information for correlation matrix. Moreover, after investigating the directions of eigenvector elements, we find that only in maximum eigenvalue vectors the elements are all same signed through the years. The contribution of individual stocks to the principal correlation proportion are all on the same direction. So we propose that the maximum eigenvalue can be taken as an indicator for the degree of collective behavior. In the turbulent time of the market, the maximum eigenvalues reach the record high level. The Spearman correlation coefficients between market volatility and the maximum eigenvalue series are all significantly positive across different windows. But the positive correlation effect turns weak in context of highest and lowest level of market volatility. The trough of varying correlation between market volatility and collective behavior indicator enable us to describe the trend changes of market risk. After defining a stability rate, we find the collective behavior of stocks tends to be more stable and homogeneous in the period of crisis than regular time.

Last, the existing literature usually adopts more complicated methodology (Liu, et al., 2011 and Tse, et al., 2010). We in contrast investigate the underlying mechanism of eigenvalues and eigenvectors. The proposed measure of collective behavior is rooted in the statistical properties of correlation matrix. The evidence provides the fundamental reasons for existing patterns observed, for example, disrupted scale-free network. The high correlated equities returns in the period of crisis will harm the interest of investors who use the traditional portforlio thery for asset pricing.

\section{Acknowledgment}

The work was partially supported by National Natural Science Foundation of China (No.11271368), Beijing Philosophy and Social Science Foundation Grant(No.12JGB051), Project of Ministry of Education supported by the Specialized Research Fund for the Doctoral Program of Higher Education of China (Grant No. 20130004110007), The Key Program of National Philosophy and Social Science Foundation Grant (No. 13AZD064), Fundamental Research Funds for the Central Universities and the Research Fundsof Renmin University of China (No.10XNK025) and China Statistical Research Project (No. 2011LZ031). We thank Prof. Chen and other members in the research group of Institute of Theoretical Physics, CAS, for the helpful discussion.

\section{References}

[1] Agmon, T. (1972). The relations among equity markets: a study of share price comovements in the United States, United Kingdom, Germany and Japan. The Journal of Finance 27(4), 839-855. 
[2] Aneja, Y. P., Chandra, R., Gunay, E.(1989). A Portfolio Approach to Estimating the Average Correlation Coefficient for the Constant Correlation Model. The Journal of Finance 44(5), 1435-1438.

[3] Arouria, M. E. H., Bellalahb, M., and Nguyenc, D. K. (2010). The co-movements in international stock markets: new evidence from Latin American emerging countries. Applied Economics Letters 17(13), 1323-1328.

[4] Bekaert, G., Hodrick, R. J., and Zhang, X. (2009). International stock return co-movements. The Journal of Finance 64(6), 2591-2626.

[5] Bouchaud, J. P.,Potters, M.(2003). Theory of financial risk and derivative pricing: from statistical physics to risk management, Cambridge: Cambridge University Press.

[6] Buraschi, A., Porchia, P., Trojani, F. (2010). Correlation risk and optimal portfolio choice. The Journal of Finance 65(1), 393-420.

[7] Chesnay, F., and Jondeau, E. (2001). Does correlation between stock returns really increase during turbulent period? Economic Notes 30(1), 53-80.

[8] Dragulescu, A., Yakovenko, V. M.(2000). Statistical mechanics of money. The European Physical Journal B 17, 723-729.

[9] Driessen, J., Maenhout, P. J., and Vilkov, G.(2013). Option Implied Correlations and the Price of Correlation Risk. Advanced Risk \& Portfolio Management Paper. Available at SSRN: http://ssrn.com/abstract=2166829 or http://dx.doi.org/10.2139/ssrn.2166829

[10]Goldstein, M. L., Morris, S. A., and Yen, G. H. (2004). Problems with fitting to the powerlaw distribution. The European Physical Journal B 41, 255-258.

[11]Hollander, M., and Wolfe, D.A.(1973). Nonparametric Statistical Methods, Wiley: New York.

[12]Jea, R., Su, C. -T., Lin, J. -L. (2005). Time Aggregation Effect on the Correlation Coefficient: Added-Systematically Sampled Framework. Journal of the Operational Research Society 56, 1303-1309.

[13]Laloux, L., Cizeau, P., Potters, M., and Bouchaud, J. (2000). Random matrix theory and financial correlations. International Journal of Theoretical and Applied Finance 03(03), 391-397. 
[14]Liu, J., Tse, C. K., and He, K.(2011). Fierce stock market fluctuation disrupts scalefree distribution, Quantitative Finance 11(6), 817-823.

[15]Mantegna, R.N., Stanley, H.E. (1999). Introduction to Econophysics: Correlations and Complexity in Finance, Cambridge: Cambridge University Press.

[16]Maskawa, J., Souma, W., Fukuda, K. (2009). Large Correlations and the Principal Componentsin Stock Markets, Proceedings of the $9^{\text {th }}$ Asia-Pacific Complex Systems Conference, 7-11.

[17]Petersen, A. M., Wang, F., Havlin, S., and Stanley, H. E. (2010). Market dynamics immediately before and after financial shocks: Quantifying the Omori, productivity, and Bath laws. Physical Review E 82, 036114.

[18]Plerou, V., Gopikrishnan, P., Rosenow, B., Amaral, L.A.N., and Stanley, H.E. (2000). A random matrix theory approach to financial cross-correlations. Physica A 287, 374-382.

[19]Plerou,V., Gopikrishnan,P., Rosenow, B., Amaral, L.A.N., and Stanley, H.E. (2001). Collective behavior of stock price movements-a random matrix theory approach. Physica A 299, 175-180.

[20]Podobnik, B., Fu, D. F., Stanley, H. E. and Ivanov, P. Ch. (2007). Power-law autocorrelated stochastic processes with long-range cross-correlations. The European Physical Journal B 56, 47-52.

[21]Podobnik, B., Fu, D. F., Stanley, H. E., and Ivanov, P. Ch. (2009). Quantifying crosscorrelations using local and global detrending approaches. The European Physical Journal B 71, 243-250.

[22]Podobnik, B., Horvati'c, D., Tenenbaum, J.N. and Stanley, H.E. (2009). Asymmetry in power-law magnitude correlations. The European Physical Journal B 80, 015101.

[23]Podobnik, B., Wang, D., Horvati'c, D., Grosse,I., and Stanley, H.E.(2010). Time-lag crosscorrelations in collective phenomena. Europhysics Letters 90, 68001.

[24]Polleta, J. M., and Wilson, M. (2010). Average correlation and stock market returns. The Journal of Financial Economics, 96, 364-380.

[25]Sias, R. W. (2004), Institutional herding. Review of Financial Studies, 17, 165-206.

[26]Sharifi, S., Crane, M., Shamaie, A., and Ruskin, H.(2004). Random matrix theory for portfolio optimization: a stability approach. Physica A 335(3-4), 629-643. 
[27] Sornette, D. (2002). Why Stock Markets Crash: Critical Events in Complex Financial Systems, Princeton: Princeton University Press.

[28]Struart, A., Ord, K., and Arnold, S. (1999). Kendall's Advanced Theory of Statistics, Arnold: London.

[29]Tse, C.K., Liu, J., and Lau, F.C.M. (2010). A network perspective of the stock market. Journal of Empirical Finance 17(4), 659-667.

[30]Wermers, R. (1999), Mutual fund herding and the impact on stock prices. Journal of Finance, 54, 581-622.

Received December 3, 2013; accepted February 22, 2014.

Zhaoyuan Li

Department of Statistics and Actuarial Science

The University of Hong Kong, Hong Kong

Rm 518, Meng Wah Complex

zyli12@hku.hk

Sibo Liu

Department of Finance and Insurance Lingnan University, Hong Kong

Simon and Eleanor Kwok Building, Lingnan University, 8 Castle Peak Road, Tuen Mun sibo@hku.hk

Maozai Tian

Center for Applied Statistics, School of Statistics Renmin University of China

Beijing, 100872, China

mztian@ruc.edu.cn 
